$4-1-1998$

\title{
Immunochemical and Immunocytochemical Identification of a Myosin Heavy Chain Polypeptide in Nicotiana Pollen Tubes
}

\author{
X. J. Tang \\ Beijing Agricultural University \\ P. K. Hepler \\ Beijing Agricultural University
}

Stylianos P. Scordilis

Smith College, sscordil@science.smith.edu

Follow this and additional works at: https://scholarworks.smith.edu/bio_facpubs

Part of the Biology Commons

\section{Recommended Citation}

Tang, X. J.; Hepler, P. K.; and Scordilis, Stylianos P., "Immunochemical and Immunocytochemical Identification of a Myosin Heavy Chain Polypeptide in Nicotiana Pollen Tubes" (1998). Biological Sciences: Faculty Publications, Smith College, Northampton, MA.

https://scholarworks.smith.edu/bio_facpubs/148

This Article has been accepted for inclusion in Biological Sciences: Faculty Publications by an authorized administrator of Smith ScholarWorks. For more information, please contact scholarworks@smith.edu 


\title{
Immunochemical and immunocytochemical identification of a myosin heavy chain polypeptide in Nicotiana pollen tubes
}

\author{
XIAOJING TANG ${ }^{1,2,3}$, PETER K. HEPLER ${ }^{2}$ and STYLIANOS P. SCORDILIS ${ }^{3}$ \\ 'Department of Biochemistry, Beijng Agricultural Unrerstty, Beijing. China \\ ${ }^{2}$ Botany Department, Unversity of Massachusetts, Amherst, MA 01003, USA \\ ${ }^{3}$ Department of Biological Sciences, Smith College, Northampton, MA 01063, USA
}

\section{Summary}

A myosin heavy chain polypeptide has been identified and localized in Nicotiana pollen tubes using monoclonal anti-myosin antibodies. The epitopes of these antibodies were found to reside on the myosin heavy chain head and rod portion and were, therefore, designated anti-S-1 (myosin S-1) and antiLMM (light meromyosin). On Western blots of the total soluble pollen tube proteins, both anti-S-1 and anti-LMM label a polypeptide of approximately $175000 M_{\mathrm{r}}$. Immunofluorescence microscopy shows that both antibodies yield numerous fluorescent spots throughout the whole length of the tube, often with an enrichment in the tube tip. These fluor-

escent spots are thought to represent vesicles and/or organelles in the pollen tubes. In addition to this common pattern, anti-S-1 stains both the generative cell and the vegetative nuclear envelope. The different staining patterns of the nucleus between anti-S-1 and anti-LMM may be caused by some organization and/or anchorage state of the myosin molecules on the nuclear surface that differs from those on the vesicles and/or organelles.

Key words: myosin, Western blotting, immunofluorescence, cytoplasmic streaming, nuclear migration.

\section{Introduction}

Cytoplasmic streaming in plant cells is thought to be caused by an actin-myosin system (see review by Staiger \& Schliwa, 1987). A good example of such a system in higher plants is the pollen tube, which exhibits vigorous cytoplasmic streaming. Actin in pollen tubes has been well-documented, especially in those of Nicotiana alata (see review by Staiger \& Schliwa, 1987; Lancelle \& Hepler, 1989; Tang et al. 1989). In contrast, our knowlege of pollen tube myosin is rather poor. The direct evidence for the existence of myosin is limited. Further, how myosin may mediate cytoplasmic streaming and/or nuclear migration remains obscure.

The model of the interaction between actin and myosin in non-muscle cells might well differ from that in muscle cells. In the latter, myosin molecules assemble into thick filaments that co-operatively interact with actin thin filaments to produce the contractile force; while in the former, myosin monomers or oligomers may interact with actin filaments, as myosin exists in much lower abundance than in muscle cells and no thick filaments have been found in vivo. Recently, in vitro experiments showed that both skeletal muscle myosin-coated beads (Sheetz \& Spudich, 1983) and isolated organelles from Lilium pollen tubes (Kohno \& Shimmen, 1988) can move actively along natural Chara actin bundles under proper conditions. It has been suggested that the membranebound vesicles and organelles, including nuclei, which move along the actin cables in streaming cells, might be coated with myosin molecules. Here we report the identification of myosin in pollen tubes of Nicotiana alata and show an immunological staining pattern consistent with the idea that the organelles (and/or vesicles) and the nuclei are indeed coated with myosin molecules.

\section{Materials and methods}

SDS-polyacrylamide gel electrophoresis (SDS-PACE) and Western blotting

Pollen tubes were prepared as described previously (Tang et al. 1989). The pollen tube proteins soluble in high ionic strength were extracted for $30 \mathrm{~min}$ in a buffer containing $0.5 \mathrm{M}-\mathrm{KCl}$, $15 \mathrm{~mm}$-Tris- $\mathrm{HCl}, \mathrm{pH} 7 \cdot 5,1 \mathrm{~mm}$-EDTA, $5 \mathrm{~mm}$-dithiothreitol (DTT), including $0 \cdot 1 \mathrm{~mm}$-phenylmethylsulphonyl fluoride (PMSF), $1 \mu \mathrm{M}$-antipain, $1 \mu \mathrm{M}$-pepstatin A, $1 \mu \mathrm{g} \mathrm{ml}{ }^{-1}$ leupeptin, $10 \mu \mathrm{g} \mathrm{ml}^{-1}$ L-1-tosylamide-2-phenyl-ethylchloromethyl ketone (TPCK), and $10 \mu \mathrm{g} \mathrm{ml}^{-1} N$-benzoyl-L-arginine ethyl ester hydrochloride (BAEE) as proteolytic enzyme inhibitors. The proteins were then precipitated with acetone and run on $7.5 \%$ SDS-PAGE slab gels (Laemmli, 1970). Following electrophoresis, the proteins were transfered electrophoretically onto 


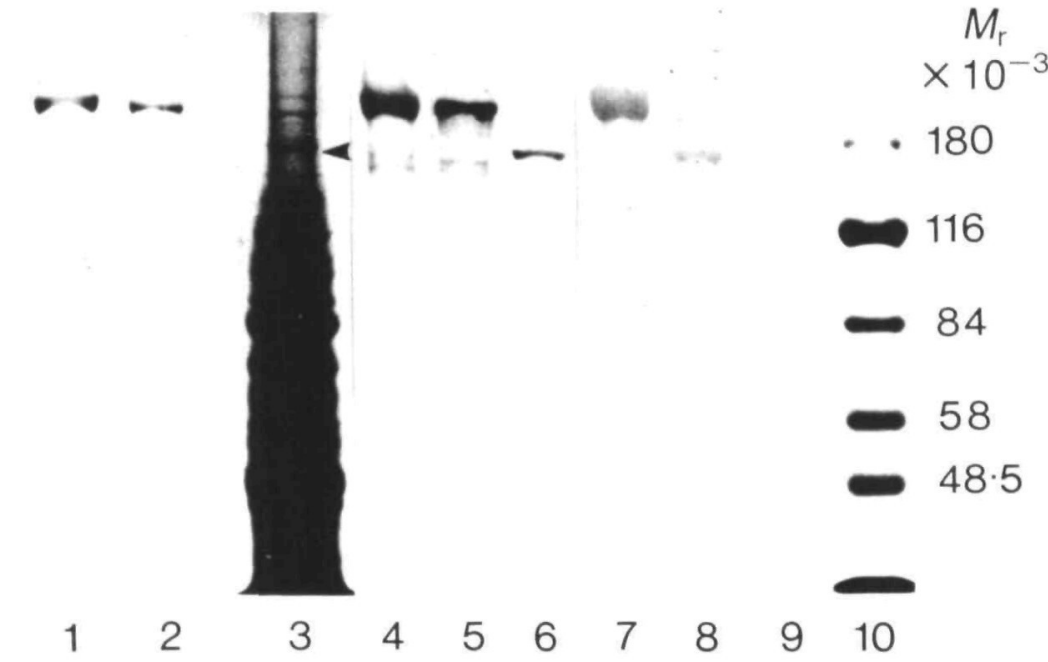

Fig. 1. SDS-polyacrylamide gel electrophoresis and Western blot analysis. Lanes 1, 2 and $3: 7.5 \%$ SDS-polyacrylamide gels of bovine skeletal muscle myosin (lane 1), rabbit skeletal muscle myosin (lane 2) and overloaded pollen tube soluble protein extract (lane 3) stained with Coomassie Blue. Lanes 4-9: nitrocellulose blots of 7.5\% SDSpolyacrylamide gels containing bovine skeletal muscle myosin (lane 4), rabbit muscle myosin (lanes 5 and 7) and pollen tube soluble protein extract (lanes 6, 8 and 9) loaded as in lane 3. Lanes 4-6 were stained with anti-LMM antibody, and lanes 7 and 8 were stained with anti-S-1 antibody. Note that a polypeptide of about $175000 M_{\mathrm{r}}$ in the pollen tube soluble protein extract is labelled by both anti-LMM and anti-S-1, and this band is also visible on the heavily loaded Coomassie Bluestained gel (arrowhead in lane 3 ). As a control strip, lane 9 was incubated only with the peroxidase-tagged secondary antibody. Molecular weight markers are indicated on the right $\left(\times 10^{-3}\right)$.

nitrocellulose (Towbin et al, 1979). The nitrocellulose sheets were then blocked with a solution of Tris-buffered saline (TBS) containing $2 \%$ bovine serum albumin (BSA) and $0.1 \%$ Triton $\mathrm{X}-100$, overnight at room temperature, and incubated in antifast skeletal muscle myosin (anti-S-1) or anti-pan cytoplasmic myosin (anti-LMM) (RPN.1167 and RPN.1169, respectively; Amersham Corp.) appropriately diluted in the above blocking solution. These designations are explained in Results. Following washes in TBS containing $0.05 \%$ Tween 20 , the primary antibodies were detected using the peroxidase-tagged biotin-streptavidin system (Amersham Corp.) for anti-S-1 and peroxidase-tagged anti-mouse IgM (Sigma Chemical Co.) for anti-LMM. Colour development was done in $1 \mathrm{mg} \mathrm{ml}^{-1} \mathrm{di}$ aminobenzidine, $0.0075 \% \mathrm{H}_{2} \mathrm{O}_{2}$ in TBS. In controls we omitted the primary antibodies.

Either rabbit and/or bovine skeletal muscle myosins (Sigma Chemical Co.) were run on the gels as standard myosins. To map grossly the epitopes to which the antibodies bind, rabbit skeletal muscle myosin subfragment-1 (S-1) and light meromyosin (LMM) (Sigma Chemical Co.) were also run and processed as described above.

\section{Immunofluorescence microscopy}

Pollen tubes were germinated as described previously and fixed in cold acetone $\left(-20^{\circ} \mathrm{C}\right)$ for $25 \mathrm{~min}$. Following cell wall digestion (Tang et al. 1989), the pollen tubes were preincubated in TBS with $1 \%$ BSA and $10 \%$ normal goat serum to block non-specific binding sites. The pollen tubes were then incubated in either anti-S-1 or anti-LMM, appropriately diluted in TBS, with $1 \% \mathrm{BSA}$ and $5 \%$ normal goat serum. After three washes, FITC-tagged biotin-streptavidin (Amersham Corp.) or FITC-tagged anti-mouse IgM (Sigma Chemical Co.) was applied. For colocalızing nuclei and myosin, thodamine-tagged anti-mouse IgM (Sigma Chemical Co) was used as the myosin-detection system, and 4,6-diamidino-2phenylendiamine (DAPI) (Sigma Chemical Co.) was used to stain DNA (Parke et al. 1986). Controls were prepared by omitting the primary antibodies or by applying primary antibodies absorbed with rabbit skeletal muscle myosin (Sigma Chemical Co.). Fluorescence was visualized and photographed using a Reichert microscope.

\section{Results}

\section{Westem blotting}

Western blots of total high ionic strength extractable

pollen tube proteins show that a polypeptide of about $175000 M_{\mathrm{r}}$ is labelled by both anti-myosin antibodies, anti-S-1 and anti-LMM. Coomassie Blue-stained bovine (lane 1) and rabbit (lane 2) skeletal muscle myosin heavy chain standards $\left(210000 M_{\mathrm{r}}\right)$ are shown on the SDSpolyacrylamide gels in Fig. 1, as well as a gel of a heavily loaded pollen tube extract (lane 3). Western blots of these proteins are shown probed with the anti-LMM antibody (Fig. 1, lanes 4-6, respectively). These results demonstrate that a single band in the pollen tube extract binds the anti-LMM antibody (Fig. 1, lane 6) and that this corresponds to a band of about $175000 M_{\mathrm{r}}$ in the Coomassie Blue-stained gel (lane 3, arrowhead). This same pollen tube band is bound by the anti-S-1 antibody (Fig. 1, lane 8). Standard rabbit skeletal muscle myosin stained with anti-S-1 is also shown (Fig. 1, lane 7). As a control for non-specific staining, a Western blot of the pollen tube extract was probed under the same conditions as all the other blots, but without any primary antibody. No immunological staining was observed (Fig. 1, lane 9).

The monoclonal antibodies used in this study were uncharacterized as to their binding domains in the myosin heavy chain. To map grossly the binding epitopes of the anti-fast skeletal muscle myosin and anti-pancytoplasmic myosin, Western blots of myosin heavy chain and its proteolytic fragments were probed with both antibodies. The SDS-polyacrylamide gel staining patterns of rabbit skeletal muscle myosin heavy chain (lane 1), subfragment-1 (S-1, lane 2) and light meromyosin (LMM, lane 3) are shown in Fig. 2. Western blots of the intact heavy chain (Fig. 2, lane 4) and LMM (lane 5) are shown probed with the monoclonal anti-pan cytoplasmic myosin antibody. Note that this antibody recognizes a domain in the LMM or tail region of the myosin molecule. This antibody did not bind to S-1 (data not shown). Hence, we have designated this antibody antiLMM to correspond to its binding epitope. The anti-fast skeletal muscle myosin monoclonal antibody bound to the intact heavy chain (Fig. 2, lane 6) and to the S-1 fragment of myosin (lane 7). This antibody did not bind to LMM (data not shown). Therefore, we designated this antibody anti-S-1 to indicate its binding domain in the head portion of the intact myosin molecule. While both of 


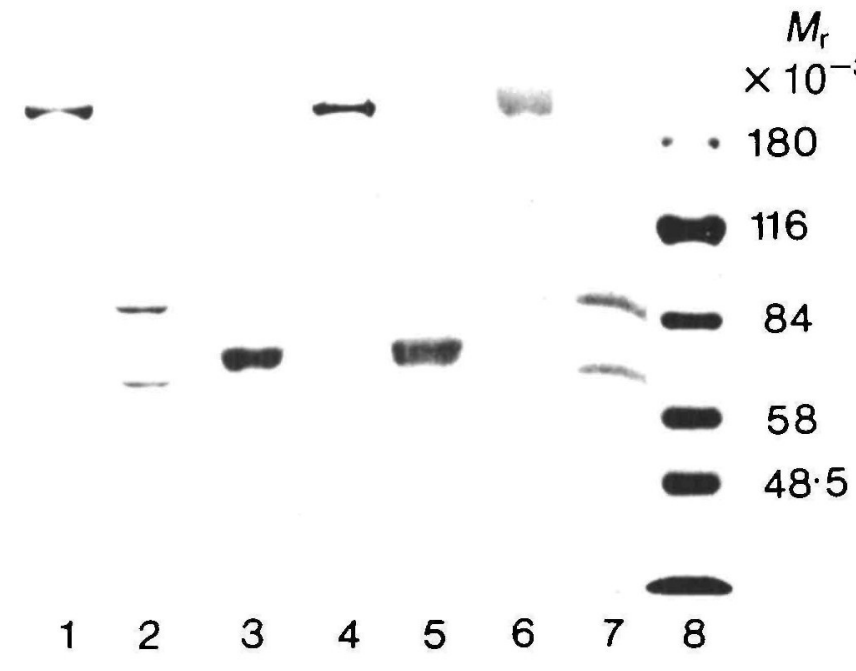

Fig. 2. SDS-polyacrylamide gel electrophoresis and Western blot analysis of intact rabbit skeletal muscle myosin, subfragment-1 (S-1) and light meromyosin (LMM) for grossly mapping the epitopes of the anti-LMM and anti-S-1 antibodies. Lanes 1-3: 7.5\% SDS-polyacrylamide gel of the intact myosin (lane 1), S-1 (lane 2) and LMM (lane 3) stained with Coomassie Blue. Lanes 4-7: nitrocellulose blots of $7.5 \%$ SDS-polyacrylamide gels with the whole myosin molecule (lanes 4 and 6). LMM (lane 5) and S-1 (lane 7) stained with anti-LMM (lanes 4 and 5), and anti-S-1 (lanes 6 and 7). The molecular weight of S-1 is about 95000 and the lower band is the fragment of S-1. Molecular weight markers are indicated on the right $\left(\times 10^{-3}\right)$.

these antibodies will bind to the intact myosin heavy chain, they can be used to indicate grossly two different domains of the myosin heavy chain, namely the head (anti-S-1) and the tail (anti-LMM).

\section{Immunofluorescence microscopy of pollen tubes}

Both anti-LMM (Fig. 3A-C) and anti-S-1 (E-G) show significant punctate fluorescence throughout the whole length of the pollen tube. Different images can be obtained by changing the plane of focus. The fluorescent spots are somewhat variable in size; and some are obviously bigger and brighter than others (B, arrow). There seem to be more spots in the tip region than in the other portions, especially with anti-LMM (A-C). The diffuse fluorescent background partially comes from outof-focus spots.

Although the staining patterns from anti-S-1 and antiLMM are very similar with respect to the punctate fuorescence in the cytoplasm, they differ with respect to the nuclear surface. Anti-S-1, in contrast to anti-LMM, stains the vegetative nuclear envelope (arrowhead, in Fig. 3G) and the generative cell (arrow, in Fig. 3G). Double labelling with DAPI $(\mathrm{H})$ has been used to stain the nuclei and, thereby, establishes that both are positive for myosin (Fig. 3G and $\mathrm{H}$ ). With regard to the generative cell, however, we are not able to determine whether the staining emanates from the nuclear envelope or the plasma membrane.

Control cells in which primary antibodies were omitted show neither punctate fluorescence nor nuclear staining
(Fig. 3D). As a futher control on the specificity of staining, the antibodies were mixed with rabbit skeletal muscle myosin at various concentrations and applied to pollen tubes. Microscopic examination showed little or no vesicular or nuclear staining (data not shown).

\section{Discussion}

Our results show that myosin exists in Nicotiana pollen tubes and that myosin antibodies produce a markedly punctate fluorescence pattern. Further, the anti-S-1 antibody stains both the generative cell surface and the vegetative nuclear envelope. Our results provide the first direct evidence for the existence of myosin in pollen tubes, although plant myosin has been reported in a few species including the alga Nitella flexilis (Kato \& Tonomura, 1977), and in the higher plants Cucurbita moschata (Yen \& Shih, 1965), Egeria densa (Ohsuka \& Inoue, 1979), Lycopersicon esculentum (Vahey \& Scordilis, 1980; Vahey et al. 1982), Allium cepa (Parke et al. 1986; Tang \& Yen, 1988), Luffa cylindrica (Yen et al. 1986) and Heracleum sosnowskye (Turkina et al. 1987). However, given the ubiquity of cytoplasmic streaming and the likelihood that it is caused by an actin-myosin system, it is anticipated that myosin occurs throughout all plants.

On Western blots of total soluble pollen tube proteins, both anti-S-1 and anti-LMM recognize a polypeptide of about $175000 M_{\mathrm{r}}$. This polypeptide is believed to be the heavy chain of Nicotiana pollen myosin, as it is known that both of the antibodies bind to the heavy chains of skeletal muscle myosins. Different molecular weights of myosin heavy chain have been reported in a variety of plant species. In the alga Nitella flexilis (Kato \& Tonomura, 1977) and in the higher plant Allium cepa (Parke $e t$ al. 1986), a $200000 M_{\mathrm{r}}$ myosin heavy chain has been reported, whereas $180000 M_{\mathrm{r}}$ and $100000 M_{\mathrm{r}}$ myosin heavy chains have been reported in Egeria densa (Ohsuka \& Inoue, 1979) and Lycopersicon esculentum (Vahey \& Scordilis, 1980; Vahey et al. 1982), respectively. Mini-myosin heavy chains of about $130000 M_{\mathrm{r}}$ have also been found in Acanthamoeba castellanii (Albanesi et al. 1983) and recently in mammalian cells (Hoshimaru \& Nakanishi, 1987). It appears that myosins in different species differ in their molecular weights. We believe that the $175000 M_{\mathrm{r}}$ polypeptide is the naturally occurring heavy chain of Nicotiana pollen myosin, as six proteolytic enzyme inhibitors were used in the cell disruption and extraction medium, and no bands above or below $175000 M_{\mathrm{r}}$ were detected on the Western blots. We cannot, however, rule out the possibility of other myosin isoforms being in the pollen tubes.

Immunofluorescence microscopy reveals punctate fluorescence staining throughout the pollen tube, in which the bright fluorescent spots often seem concentrated in the tip region. Although the fluorescent spots cannot be correlated precisely with any structural elements at present, on the basis of their distribution, we think that they represent the organelles and membrane-bound vesicles. Electron microscopy has shown that the organelles 

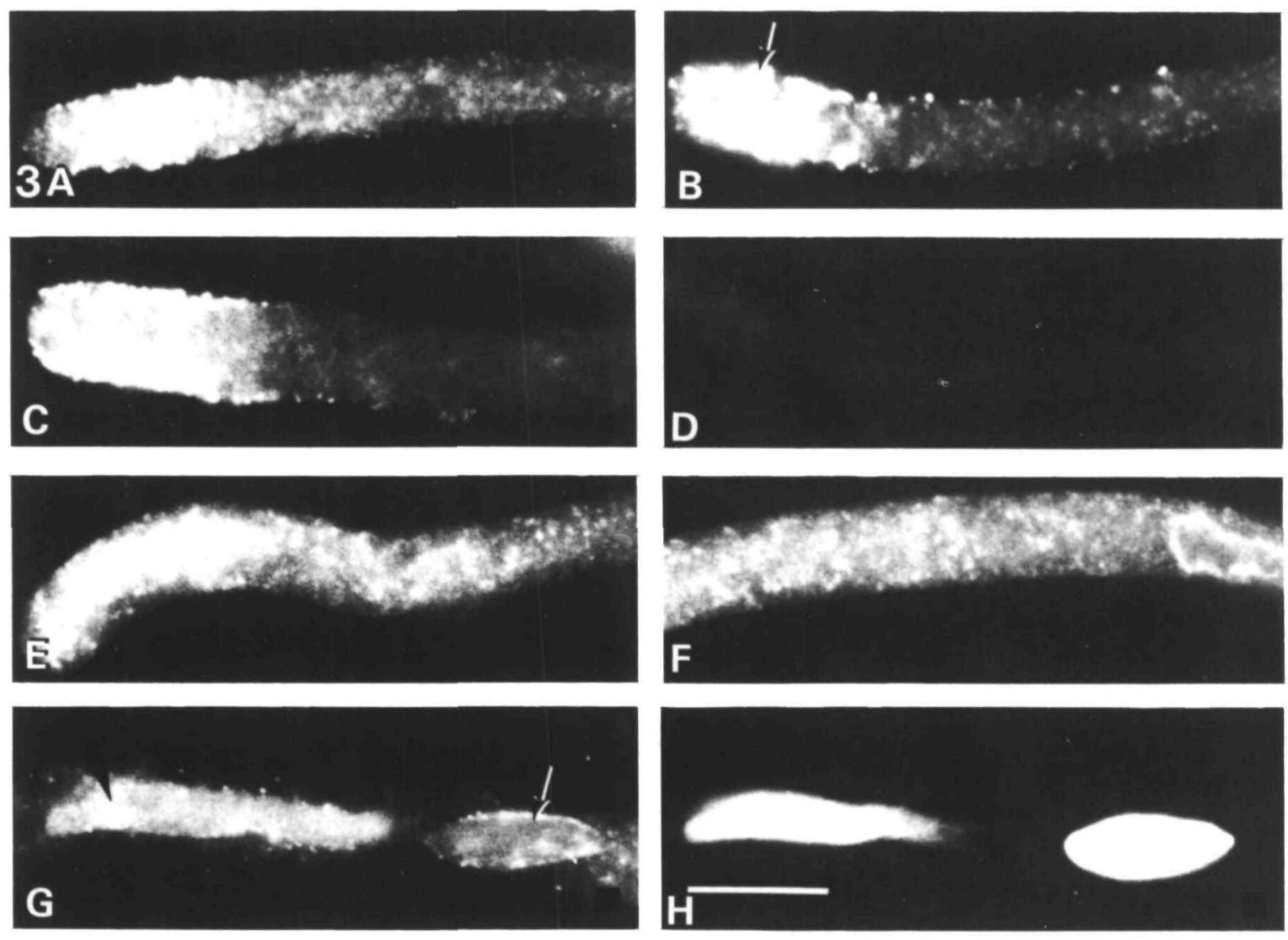

Fig. 3. Immunofluorescence micrographs of the pollen tubes staned with anti-LMM $(A-C)$ and anti-S-1 (E-G). Both antiLMM and anti-S-1 stain the presumed organelles and/or vesicles as fluorescent spots. These fluorescent spots are distributed throughout the whole length of pollen tube and they are often concentrated in the tip region, especially with anti-LMM. The fluorescent spots are variable in size and intensity; some (B, arrow) are obviously bigger and brighter than others. Anti-S-1 also stains the generative cell $(G$, arrow) and vegetative nuclear envelope $(G$, arrowhead). $H$. The same pollen tube as in $G$ stained with DAPI. D. The control pollen tube that has been prepared omitting the primary antibodies. Bar, $5 \mu \mathrm{m}$.

and vesicles are distributed throughout the pollen tube and that vesicles are concentrated in the tip region (Lancelle et al. 1987). Recently, Kohno \& Shimmen (1988) reported that isolated organelles from Lilium pollen tubes can move actively along Chara actin cables in the presence of ATP and low $\mathrm{Ca}^{2+}$ concentration, and suggested that myosin molecules are associated with these organelles. Our results support this hypothesis, although further immuno-gold localization at the electron microscopic level is needed to resolve the question more completely.

An interesting difference between anti-S-1 and antiLMM is that the former stains the generative cell surface and the vegetative nuclear envelope, as well as the presumed organelles and/or vesicles. Since both anti-S-1 and anti-LMM recognize the same myosin heavy chain of the pollen tubes, the different staining patterns may be caused by the different micro-environments of the nuclear and organellar myosins. A simple explanation may be derived from the observation that different portions of the myosin heavy chain are being recognized. Our results show that one of the antibodies (anti-LMM) binds to the rod and the other (anti-S-1) binds to the head of the myosin molecule. For myosin anchorage, the rod portion of myosin may be deeply embedded in the generative cell membrane or the vegetative nuclear envelope and, thus, inaccessible to anti-LMM. The head, that is, the enzymic portion, however, must be exposed for interaction with the actin filaments, and therefore it can be stained by anti-S-1. The myosin on vesicles and organelles may be associated with the membranes in a different way, which results in the exposure of epitopes for both anti-S-1 and anti-LMM. Since the sequence of the pollen tube myosin heavy chain is not known and since it may have diverged considerably from that of the rabbit skeletal muscle, we suggest that it may contain a hydrophobic domain(s) that could help secure it into a membrane. In this regard it may be pertinent to note that the envelopes of the vegetative and generative nuclei are double membranes, as is the plasma membrane surrounding the generative cell (Cresti et al. 1987). Myosin binding to these complex double-membrane systems may require a more extensive interaction than is found on single membranes of vesicles and also that this binding effectively obscures the LMM portion of the myosin molecules rendering it inaccessible to the anti-LMM antibody.

The mechanism of nuclear migration in the tipgrowing cells is poorly understood, although microtubules have been implicated in germinating fern spores of Onoclea sensibilis (Vogelmann et al. 1981), the moss 
Physcomitrella patens (Doonan et al. 1986), legume root hair cells (Lloyd et al. 1987) and Funaria hygrmmetrica protonemata (Wacker et al. 1988). In contrast, F-actin has been shown to be involved in basipetal migration of the nucleus in legume root-hair cells (Lloyd et al. 1987). The pollen tube is a type of fast tip-growing cell, in which the nuclei migrate along the tube during elongation. The myosin on the generative cell and vegetative nuclear surfaces might participate in nuclear migration. In this regard it is important to note that nuclear migration is unidirectional and very slow (about $1 \mu \mathrm{m} \mathrm{min}^{-1}$ ), while cytoplasmic streaming is bidirectional and fast (about $\left.1 \mu \mathrm{m} \mathrm{s}^{-1}\right)$. If both types of motion are caused by the actin-myosin system then there must be some type of compartmentation or differentiation that allows the vesicles and organelles to move rapidly and bidirectionally past the slow and tipward-moving vegetative nuclear, generative cell complex. Our observations, showing that the generative cell and vegetative nucleus are stained by anti-S-1 and not anti-LMM, suggest that the myosin is organized differently on the nuclei than it is on the organelles and vesicles, and that as a result it could impart a different form of motility. There may, of course, be some difference in the regulation of the myosin that could account for the various motility patterns; for example, different light chains, or the presence of myosin isoforms.

Another possible function of the nuclear myosin may be in mediating nucleocytoplasmic transport. The staining of the nuclear surface by myosin antibodies has also been reported in some non-tip-growing cells like yeast (Scordilis et al. unpublished), rat liver (Schindler \& Jiang, 1986), Drosophila embryo and onion root (Berrios \& Fisher, 1986). Moreover, it has been shown that myosin antibody or actin antagonists (e.g. actin antibody, cytochalasin D and phalloidin) can specifically inhibit the nucleocytoplasmic transport of fluorescently labelled dextran in rat liver cells (Schindler \& Jiang, 1986).

In conclusion, a myosin heavy chain polypeptide has been identified immunochemically in an extract of Nicotiana pollen tubes. Immunofluorescence localization of this protein shows an extensive punctate fluorescence pattern as well as staining of the generative cell and vegetative nuclear envelope. Immuno-gold electron microscopy will be useful in future work for revealing the organelles that the fluorescent spots represent.

We are very grateful to Professer L. Yen, Beijing Agricultural University, for his encouragement and valuable discussion. We thank Dr G. Scordilis, Smith College, for a critical reading of the manuscript. We also thank Ms S. Lancelle, U. MA, and Ms D. Anable, Smith College, for helpful discussions. This work was supported by NSF grant DCB:87-02057 to P. K. Hepler, by NIH grant GM40048 and the Blakeslee Genetics Fund to S. P. Scordilis, and partially by a grant from the Basic Sciences Foundation, Chinese Academy of Science to L. Yen.

\section{References}

Albanesi, J. P., Hammer, J. A. III \& Korn, E. D. (1983). The interaction of $\mathrm{F}$-actin with phosphorylated and unphosphorylated myosin IA and IB from Stcanthamoeba castellanii. 7. biol. Chem. 258, $10176-10181$.
Berrios, M. \& Fisher, P. A. (1986). A myosin heavy chain-like polypeptide is associated with the nuclear envelope in higher eukaryotic cells. J. Cell Bıol. 103, 711-724.

Crest, M., Lancelle, S. A. \& Hepler, P. K. (1987). The structure of the generative cell wall complex after freeze substitution in pollen tubes of Nicotiana and Impatiens. Y. Cell Sci. 88, 373-378.

Doonan, J. H., Jenkins, G. I., Cove, D. J. \& Lloyd, C. W. (1986). Microtubules connect the migrating nucleus to the prospective division site during side branch formation in the moss, Physcomitrella patens. Eur. 7. Cell Biol. 41, 157-164.

Hoshimaru, MI. \& Nakanishi, S. (1987). Identification of a new type of mammalian myosin heavy chan by molecular cloning. f. biol. Chem. 262, 14625-14632.

Kato, T. \& Tonomura, Y. (1977). Identification of myosin in Nitella flexilis. J. Biochem. 82, 777-782.

Konno, T. \& Shimmen, T. (1988). Accelerated sliding of pollen tube organelles along Characeae actin bundles regulated by $\mathrm{Ca}^{2+}$ J. Cell Biol. 106, 1539-1543.

LAEMMLI, U. (1970). Cleavage of structural proteins during the assembly of the head of bacteriophage T4. Nature, Lond. 227, 680-685.

Lancelle, S. A., Crest1, M. \& Hepler, P. K. (1987).

Ultrastructure of the cytoskeleton in freeze-substituted polien tubes of Nicotiana alata. Protoplasma 140, 141-150.

Lancelle, S. A. \& HePler, P. K. (1989). Cytochalasin-induced ultrastructural alteration in Nicotiana pollen tubes. Protoplasma (Suppl. 2), 65-75.

Lloyd, C. W., Pearce, K. J., Rawlins, D. J., Ridge, R. W. \& SHAW, P. J. (1987). Endoplasmic microtubules connect the advancing nucleus to the tip of legume root hairs, but F-actin is involved in basspetal migration. Cell Motil. Cytoskel. 8, 27-36.

Ohsuka, K. \& Inoue, A. (1979). Identification of myosin in a flowering plant Egena densa. J. Biochem. 85, 375-378.

Parke, J., Miller, C. \& Anderton, B. H. (1986). Higher plant myosin heavy-chain identıfied using a monoclonal antıbody. Eur. $\mathcal{Y}$ Cell Biol. 41, 9-13.

SCHINDLER, M. \& JlaNG, L. (1986). Nuclear actin and myosin as control elements in nucleocytoplasmic transport. J. Cell Biol. 102, 859-862.

SHEETZ, N. \& SPUdich, J. M. (1983). Movement of myosin-coated fluorescent beads on actin cables in vitro. Nature, Lond. 303, $31-35$.

Staiger, C. J. \& Schliwa, M. (1987). Actin localization and function in higher plants. Protoplasma 141, 1-12.

Tang, X., Lancelle, S. A. \& Hepler, P. K. (1989). Fluorescence microscopic localization of actin in pollen tubes: Comparison of actın antıbody and phallordin staining. Cell Motil. Cytoskel. (in press).

TANG, X. \& YEN, L. (1989). Immunochemical identification of myosin and actin in the bulb of Allium cepa. Acta Biol. exp. sin. 21 (in press).

Towbin, H., Staehelin, T. \& Gordon, T. (1979). Electrophoretic transfer of proteins from polyacrylamide gel to nitrocellulose sheets: Procedure and some applications. Proc, natn. Acad. Sci. U.S.A. 76, 4350-4354.

Turkina, M. V., Kulikova, A. L., Sokolov, O. I., Bogatyrev, v. A. \& Kursanov, A. L. (1987). Actin and myosin filaments from the conducting tissues of Heracleum. Pl. Physiol. Biochem. 25, $689-696$.

VAHEY, M. \& SCORDILIS, S. P. (1980). Contractile proteins from tomato. Can. Y. Bot. 58, 797-801.

Vahey, M., Titus, M., Trautwein, R. \& Scordilis, S. P. (1982). Tomato actin and myosin: Contractile protens from a higher land plant. Cell Motil. 2, 131-147.

Vogelmann, Th. C., Bassel, A. R. \& Miller, J. H. (1981). Effects of microtubule-inhibitors on nuclear migration and Rhizoid differentiation in germnating fern spores (Onoclea sensibilis). 
Protoplasma 109, 295-316.

WaCker, I., Quader, H. \& SChNePF, E. (1988). Influence of the herbicide oryzalin on cytoskeleton and growth of Funaria hygrometrica protonemata. Protoplasma 142, 55-67.

YEN, L. \& SHIH, T. (1965). The presence of a contractile protein in higher plants. Scientia Sinica 14, 601-608.
Yen, L., Wang, X., Teng, X., Ma, Y. \& LiU, G. (1986). Actin and myosin in pollens and their role in the growth of pollen tubes. Kexue Tongbao (Science Bulletin) 31, 267-272.

(Received 9 September 1988 - Accepted, in revised form, 10 January 